\title{
Análise Crítica das DCN à Luz das Diversidades: Educação Médica e Pandemia da Covid-19
}

\author{
Critical Analysis of the National Curriculum Guidelines in the Light of Diversities: Medical Training and the \\ Covid-19 Pandemic
}

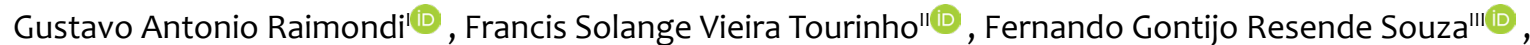

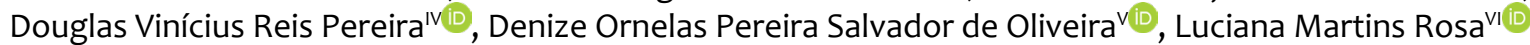

\section{RESUMO}

Introdução: Os movimentos sociais, organizados em torno da reforma sanitária, contribuíram para a institucionalização do processo de formação em saúde no país que deve estar em consonância com as reais necessidades de saúde da população e, dessa forma, promover a inclusão e equidade na perspectiva da social accountability. Isso se torna ainda mais importante no contexto da pandemia da Covid-19, já que se vivencia uma nova prioridade de saúde. Entretanto, o que se tem observado ao longo da pandemia da Covid-19 é um despreparo profissional para um cuidado integral em saúde que considere as pessoas e comunidades historicamente invisibilizadas.

Objetivo: Analisar criticamente as questões das diversidades em relação às Diretrizes Curriculares Nacionais para os Cursos de Graduação em Medicina (DCN).

Desenvolvimento: Por meio de três blocos temáticos - "Análise comparativa das DCN de 2001 e 2014 na perspectiva da diversidade","Como podemos problematizar as questões da diversidade a partir das DCN de 2014?" "As DCN de 2014 e o que precisamos tornar mais evidente na busca pela diversidade no ensino médico" -, aprofundou-se o debate crítico e reflexivo sobre a educação médica a partir da perspectiva de diversidade. Constatou-se a necessidade de uma articulação formativa e assistencial com as demais políticas públicas em saúde, principalmente aquelas relacionadas a populações marginalizadas.

Conclusão: A pandemia da Covid-19 mostra-se como uma oportunidade de a mídia e a sociedade como um todo olharem para as desigualdades sociais em saúde, considerarem a relevância do SUS e enfatizarem os múltiplos apontamentos contemporâneos que evidenciam que os projetos pedagógicos e os componentes curriculares dos cursos de Medicina precisam ser atualizados e se comprometer com a construção de uma proposta de ensino e cuidado em saúde que valorize a diversidade e diminuição das iniquidades em saúde.

Palavras-chave: Diversidade Cultural; Educação Médica; Covid-19; Promoção da Saúde; Vulnerabilidade em Saúde.

\begin{abstract}
Introduction: Social movements for Public Health Reform in Brazil have contributed toward the institutionalization of the health training process, to bring it in line with real public health needs, thus promoting inclusion, equality and an approach based on social accountability. With the onset of the Covid-19 pandemic this change has become even more important, as a new health priority has emerged. However, throughout the pandemic there has been an evident lack of professional preparation to provide comprehensive health care for people and communities who have historically been disregarded.
\end{abstract}

Objective: Critically analyze the issues of diversities in relation to the National Curriculum Guidelines (DCNs) for the undergraduate medicine courses.

Development: The critical and reflexive debate on Medical Education is developed from a perspective of diversity in three parts: 1: Comparative analysis of the DCNs from 2001 to 2014 in relation to the perspective of diversity; 2: How the issues of diversity can be critically questioned based on the 2014 DCNs; 3: The 2014 DCNs and what needs to be demonstrated in the search for diversity in medical education. Furthermore, the need for a connection between training and care was made explicit through the other public health policies, especially those related to marginalized groups.

Conclusion: The Covid-19 pandemic has proven to be an opportunity for the media and society as a whole to recognize social inequalities in health care and the relevance of the Unified Health System (SUS), and to highlight the multiple indications that the pedagogical projects and curricular components of Medicine courses need updating and to commit to building an educational and health care proposal that values diversity and reduces inequalities in health.

Keywords: Cultural Diversity; Medical Education; Covid-19; Health Promotion; Health vulnerability.

'Universidade Federal de Uberlândia, Uberlândia, Minas Gerais, Brasil.

"Universidade Federal de Santa Catarina, Florianópolis, Santa Catarina, Brasil.

"'Universidade Federal de Lavras, Lavras, Minas Gerais, Brasil.

IVFaculdade Ciências Médicas de Minas Gerais, Belo Horizonte, Minas Gerais, Brasil.

'Universidade Nove de Julho, São Paulo,São Paulo, Brasil.

v'Universidade Federal do Goiás, Goiânia, Goiás, Brasil.

Correspondência:

Gustavo AntonioRaimondi.

Universidade Federal de Uberlândia, Avenida Pará, 1720, bloco 2U, sala 8, câmpus Umuarama, Uberlândia, MG, Brasil. CEP: $38400-902$.

E-mail: gustavo.raimondi@gmail.com

Recebido em 11/08/20; Aceito em 24/08/20. 


\section{INTRODUÇÃO}

Os movimentos sociais, organizados em torno da reforma sanitária, contribuíram para a institucionalização do processo de formação em saúde no país, de tal maneira que a Constituição Federal estabelece, em seu artigo 200, que compete ao Sistema Único de Saúde(SUS)“ordenar a formação de recursos humanos na área de saúde" "Essa formação deve estar em consonância com as reais necessidades em saúde da população, e, sendo assim, promoveu-se a inclusão da perspectiva da social accountability (responsabilidade social) como um elemento central para o paradigma de educação na saúde para o novo milênio².

Segundo essa perspectiva, os processos formativos em saúde, de pesquisa e oferta de serviços, devem ser orientados e construídos em conjunto com as prioridades de saúde da comunidade - principalmente aquelas mais vulneráveis 3 . Além disso, o SUS traz a equidade como um de seus princípios doutrinários, de tal maneira que a política de saúde reconheça a pluralidade dos grupos populacionais e suas necessidades específicas, a fim de que sejam atendidos até o limite do que o sistema puder oferecer para todos ${ }^{4}$. Assim, a graduação em Medicina deve ter como compromisso tornar o futuro profissional capacitado para reconhecer tais diferenças entre seus pacientes, a fim de promover cuidados guiados pela equidade.

Isso se torna ainda mais importante no contexto da pandemia da coronavirus disease 2019 (Covid-19), doença causada pelo vírus Sars-Cov-2, já que se vivencia uma nova prioridade de saúde. Inicialmente, ponderava-se que uma maior vulnerabilidade à doença estaria relacionada principalmente às pessoas idosas e com comorbidades crônicas. Entretanto, ao longo da pandemia, explicitou-se que essa compreensão de vulnerabilidade deveria ser aprimorada para pessoas e comunidades com risco aumentado de exposição, adoecimento com gravidade e/ou morte. Entre esses(as) sujeitos(as) e coletivos, destaca-se aqueles social e historicamente marginalizados como as população negra e indígenas, pessoas com deficiência/diversidade funcional e os membros da comunidade de lésbicas, gays, bissexuais, travestis e transexuais (LGBT) 5 ,

Nesse sentido, o que se tem observado ao longo da pandemia da Covid-19 é um despreparo profissional para um cuidado integral em saúde que considere essas pessoas e comunidades invisibilizadas. Se, de acordo com Frenk et al.7, os currículos e documentos legais orientadores da formação em saúde precisam ir ao encontro das reais necessidades em saúde das pessoas e comunidades, a atual pandemia tem demonstrado que precisamos compreender como os processos formativos em saúde consideram as questões das vulnerabilidades e diversidades, como raça/etnicidade e identidade de gênero e sexualidade.

Assim, o presente ensaio buscará analisar criticamente as questões das diversidades em relação às Diretrizes Curriculares Nacionais para os Cursos de Graduação em Medicina $(D C N)^{8}$. Uma vez que a demanda do sistema de saúde por profissionais comformação generalista, humanitária, reflexiva ${ }^{9} \mathrm{e}$, sobretudo, atenta às necessidades de saúde de populações historicamente marginalizadas torna-se ainda mais evidente no momento atual, apresenta-se este ensaio composto de três blocos temáticos: "Análise comparativa das DCN de 200110 e 2014 na perspectiva da diversidade", “Como podemos problematizar as questões da diversidade a partir das DCN de 2014?" e "As DCN de 2014 e o que precisamos tornar mais evidente na busca pela diversidade no ensino médico".

Além disso, o percurso reflexivo provém de experiências, inquietações e debates pessoais, como educandos(as) e educadores(as), em diálogo com a literatura indexada sobre o tema. Dessa forma, pretende-se problematizar com este ensaio como os cursos de Medicina podem promover avanços em seus processos de ensino-aprendizagem em relação às diversidades - biológica, subjetiva, étnico-racial, de gênero, orientação sexual, socioeconômica, política, ambiental, cultural, ética - e aos demais aspectos que compõem o espectro da diversidade humana, como previsto nas $\mathrm{DCN}^{8}$. Ademais, esse debate poderá aprimorar a responsabilidade social da(s) escola(s) médica(s) no sentido da promoção da justiça social pela redução de iniquidades a partir da ampliação das perspectivas de cuidados, decorrentes desse aprimoramento do processo de ensino-aprendizagem.

\section{ANÁLISE COMPARATIVA DAS DCN DE 2001 E 2014 NA PERSPECTIVA DA DIVERSIDADE}

A publicação das DCN para os cursos de Medicina, no ano de 2001, representou um marco na mudança da educação médica e do ensino e da prática em saúde no Brasil. A partir de uma proposta de aprimoramento de aspectos curriculares (organização curricular, estratégias de ensino-aprendizagem e processos de avaliação) e do perfil profissional do(a) egresso(a), pretendeu-se aperfeiçoar uma formação em saúde alinhada com os desafios do século XXI, orientando os processos formativos para as reais necessidades de saúde da população e da interprofissionalidade ${ }^{11,12}$.

Dessa forma, observa-se uma explicitação da premência de as instituições educacionais e as políticas públicas em saúde e educação se reorganizarem e adaptarem a formação em saúde em conformidade com as necessidades, demandas e características da população. Esse aspecto pode ser observado, por exemplo, no artigo $3^{\circ}$ da DCN que afirma 
que o perfil do formando tem como característica o senso de responsabilidade social e o compromisso com a cidadania, a fim de promover a saúde integral do ser humano ${ }^{10}$.

Além disso, as DCN de 2001, ao proporem um currículo para a formação médica baseado em competências e habilidades, representam o início da orientação curricular para a formação generalista, humanista e direcionada à comunidade ${ }^{11}$. Portanto, observa-se uma expansão da concepção pedagógica acerca do modelo de formação em saúde, incorporando uma perspectiva biopsicossocial em contraposição a um modelo biomédico e tecnicista, caracterizado pelos "reducionismos biologicistas"13. Destacase que esses reducionismos compreendem o adoecimento a partir de uma visão predominante biológica com enfoque nos processos fisiopatológicos, em que a doença se torna o elemento central do cuidado. Já em uma perspectiva biopsicossocial, busca-se ampliar essa proposta considerando os determinantes em saúde e os elementos socioculturais como integrantes do adoecimento físico, da percepção do indivíduo em sentir-se doente e do cuidado prestado ${ }^{11}$.

Apesar de representar um grande avanço na estruturação de ensino, pesquisa e serviço em saúde, no que tange à mudança de um modelo biomédico para uma compreensão biopsicossocial, ainda se apresentavam lacunas principalmente em relação à atenção primária à saúde (APS) e à saúde coletiva ( $\mathrm{SC}$ ), e, especificamente, às Ciências Sociais e Humanas em Saúde (CSHS). Vários aspectos - do acolhimento às demandas ao cuidado integral dasaúde pautados pelo reconhecimento das dimensões, subjetividades e diversidades étnico-raciais, de gênero, sexualidade, socioeconômicas e político-histórico-culturais - mostraram-se incipientes e, muitas vezes, foram ignorados na estruturação curricular e na formação médica ${ }^{10,11}$. Dessa forma, a presença explicitada das CSHS teria a possibilidade de aprimorar as DCN de 2001 pela promoção da perspectiva de que o processo de ensino e o cuidado na saúde são fenômenos existenciais, societários, individuais e coletivos ${ }^{13,14}$.

Com a instituição do Programa Mais Médicos em 2013, por meio da Lei $\mathrm{n}^{\circ} 12.871$, que previa a reformulação das DCN dos cursos de Medicina implementadas em 2014, houve a oportunidade de aprimorar as competências, os valores e as perspectivas para a concretização do perfil do(a) egresso(a) em Medicina no âmbitos da educação e da saúde. Entre esses avanços, destacam-se o reforço à orientação da formação médica voltada para o SUS e para seu fortalecimento, e a inclusão dos eixos de atenção à saúde, gestão em saúde e educação em saúde nas competências contempladas pelos cursos de Medicina"1. Ademais, explicitou-se a importância das CSHS ao longo do processo formativo, assim como o reconhecimento e a compreensão do processo saúde-doença a partir da perspectiva da determinação social, reforçando a importância de questões relativas às diversidades na estruturação curricular e formação do perfil do(a) egresso(a) ${ }^{8}$.

Para a estruturação dessas novas diretrizes no âmbito da diversidade e com base no entendimento da determinação social do processo saúde-doença, foram incluídas as abordagens e discussões de temas transversais no currículo. Esses temas envolvem conhecimentos, vivências e reflexões sistematizados acerca dos direitos humanos, das relações étnico-raciais, da história das culturas afro-brasileira e indígena e do impacto destes nas condições de saúde dessas populações, do reconhecimento à existência da diversidade de gênero e orientação sexual, assim como as relações socioeconômicas, políticas, éticas, ambientais, culturais e demais aspectos que compõem o espectro da diversidade humana e que singularizam cada pessoa ou cada grupo social ${ }^{8}$. Todas essas adições visam aperfeiçoar a formação em saúde em relação aos aspectos da responsabilidade social como um elemento central no exercício da medicina e da promoção da justiça social, compreendida pela redução das iniquidades e desigualdades em saúde dos indivíduos e da população ${ }^{15,16}$.

\section{COMO PODEMOS PROBLEMATIZAR AS QUESTÕES DA DIVERSIDADE A PARTIR DAS DCN DE 2014?}

O livro Como e por que as desigualdades sociais fazem mal à saúde ${ }^{16}$ apresenta uma análise da determinação social no processo de saúde e doença e mostra como essas questões perpetuam sistematicamente barreiras para um cuidado integral em saúde. Dentro das possíveis barreiras, a Política Nacional de Atenção Integral à População Negra destaca a luta pela cidadania e pelo direito à saúde pautado pela equidade, uma vez que os negros têm vivenciado historicamente os efeitos do racismo institucional e das diversas violências que estruturam a sociedade brasileira ${ }^{17}$. Tal abordagem dos aspectos de raça e etnicidade no ensino e cuidado em saúde se iniciam com a relevância da obrigatoriedade de que cadastros incluam a autodeclaração dos(as) usuários(as) e produzam dados que possam ser desagregados por raça/ cor evidenciando as iniquidades em saúde. Mas, para além desse processo, é fundamental inserir como conteúdo a compreensão sobre como essas iniquidades se articulam com as práticas da atenção individual e coletiva, do ensino, da pesquisa e da gestão em saúde. Assim, a educação médica deve problematizar o racismo e suas diferentes formas de manifestação, seja pela ausência de processos de combate ao racismo institucional seja pela compreensão e postura dos profissionais, como elementos que perpetuam as iniquidades em saúde ou estabelecem processos antirracistas que visam 
à promoção da equidade racial, inclusive reconhecendo os piores indicadores relacionados ao tratamento da síndrome respiratória aguda grave e óbitos por Covid-19 nesse segmento populacional ${ }^{18}$.

No mesmo sentido inclusivo, a Política Nacional de Atenção à Saúde dos Povos Indígenas ${ }^{19}$ aponta para a importância da formação de profissionais de saúde para atuação em contextos interculturais. A compreensão de outras racionalidades de cuidado em saúde, como em cada um dos vários sistemas tradicionais indígenas, explicita a necessidade do aprimoramento de competências culturais que perpassam as práticas de cuidado individual e coletivo, de gestão e de educação em saúde. Exemplo disso é o fato de que orientações extremamente importantes no combate à Covid-19, como lavar as mãos frequentemente e utilizar máscaras e álcool em gel, são interpretadas pelos povos indígenas conforme suas especificidades culturais, exigindo comunicação culturalmente competente ${ }^{20,21}$. Ademais, materiais informativos e campanhas não alcançarão os indígenas se não estiverem disponíveis nos idiomas nativos ${ }^{22}$.

Destaca-se que a competência cultural está relacionada a um conjunto de conhecimentos, comportamentos e atitudes que promovam a compreensão das diferenças culturais e o respeito a elas $^{23}$. Ademais, é necessário ter uma postura de humildade cultural que reitera ações antiopressões do não saber e do aprender a aprender, como descrito nas $\mathrm{DCN}^{8,23}$.

Já a Política Nacional de Saúde Integral de Lésbicas, Gays, Bissexuais, Travestis e Transexuais ${ }^{24}$ problematiza as violências decorrentes das questões de identidade de gênero e orientação sexual. Observa-se que há um processo de cuidado em saúde que pressupõe a sexualidade dos(as) sujeitos(as) a partir de uma perspectiva cisgênera (que a identidade de gênero tem correspondência com o gênero atribuído à genitália) e heterossexual. Nesse sentido, a cisheterossexualidade presumida e a visibilidade seletiva em relação às pessoas acabam por promover barreiras e violências para o cuidado integral em saúde, atreladas a um discurso majoritário da "não diferença" ${ }^{25,26}$. Com isso, as práticas de ensino em saúde, como as relacionadas aos roteiros clínicos de anamnese, podem ser aprimoradas com uma perspectiva centrada no(a) usuário(a) em relação ao nome social, à identidade de gênero e à orientação sexual27. Ademais, os processos formativos precisam considerar as múltiplas vulnerabilidades que a população LGBT enfrenta e como elas podem ser exacerbadas em momentos de pandemia, como ressalta a Organização Mundial de Saúde (OMS) ${ }^{28}$.

Ainda sobre as questões de gênero e sexualidade, a Política Nacional de Atenção Integral à Saúde da Mulher²9 faz uma articulação teórico-reflexiva sobre a importância da perspectiva de gênero nas práticas de cuidado. A partir da concepções do que é gênero, podem-se ampliar as possibilidades identitárias e afetivas dos(as) sujeitos(as), as quais precisam ser incorporadas no ensino e cuidado em saúde de forma transversal, como orientado pelo $\mathrm{OMS}^{30}$. Essa perspectiva permite questionar as masculinidades hegemônicas e as interferências no processo de cuidado em saúde, como debatido pela Política Nacional de Atenção Integral à Saúde do Homem³ ${ }^{31}$ Ainda são debates que ampliam as práticas de cuidado integral relacionadas aos ciclos de vida: as Diretrizes Nacionais para a Atenção Integral à Saúde de Adolescentes e Jovens na Promoção, Proteção e Recuperação da Saúde e a Política Nacional de Saúde da Pessoa Idosa ${ }^{32,33}$. É premente, portanto, ampliar a formação médica para além das questões exclusivamente biológicas ${ }^{34}$ por meio, por exemplo, da articulação das ações e dos planos presentes nas políticas específicas, como reconhecimento de que a determinação social atravessa a prática médica socialmente referenciada.

Além do mais, o momento atual de pandemia, com a orientação para as práticas de quarentena domiciliar, distanciamento e isolamento social, explicitou, mais uma vez, a necessidade de refletir e atuar sobre a situação das populações que vivem em condições de aglomeração, principalmente nas periferias das grandes cidades, mas destacou, especialmente, o paradoxo de ofertar cuidado para a população em situação de rua. Segundo a Política Nacional para Inclusão Social da População em Situação de Rua ${ }^{35}$, essa população é marcada pela expulsão, pelo desenraizamento e pela privação. Essas questões expõem o processo de exclusão que esses(as) sujeitos(as) vivenciam e a importância de pensarmos ações e estratégias intersetoriais para promover o cuidado e os direitos humanos. Nesse sentido, práticas interprofissionais pautadas em uma formação social e humanística ${ }^{36}$ podem ser um dos possíveis caminhos para aprimorarmos o cuidado em saúde a essa população.

Ainda sobre as questões relacionadas à exclusão, a Política Nacional de Atenção Integral à Saúde das Pessoas Privadas de Liberdade no Sistema Prisional ${ }^{37}$ destaca que, apesar de as pessoas terem temporariamente o direito de ir e vir suspenso, os demais direitos fundamentais devem ser garantidos pelo Estado. Diante disso, essa política aponta para a importância de pensarmos o cuidado em saúde associado a vários pontos do sistema prisional e como os(as) custodiados(as) devem ser acompanhados(as) em relação aos princípios e às diretrizes do SUS. Entre estes, destaca-se a humanização que perpassa o comportamento profissional relacionado a uma postura que respeite as diferenças e sem a imposição de valores e crenças. Diante disso, a necessidade de aprimoramento do profissionalismo, proposto pela $\mathrm{DCN}^{8}$, 
pode abarcar o desenvolvimento de competências que tenham esse contexto ao longo dos processos formativos.

\section{AS DCN DE 2014 E O QUE PRECISAMOS TORNAR MAIS EVIDENTE NA BUSCA PELA DIVERSIDADE NO ENSINO MÉDICO}

Quando se pensa em perfil do(a) egresso(a), entende-se que há a necessidade de articulação entre os conhecimentos, as habilidades e as atitudes requeridos. $\mathrm{O}(\mathrm{a})$ egresso(a) terá que considerar não apenas as dimensões das diversidades na sua prática profissional, mas também e de forma crítica as competências pautadas no compromisso social.

De acordo com as DCN, o discente, na atenção à saúde,

[...] será formado para considerar sempre as dimensões da diversidade biológica, subjetiva, étnico-racial, de gênero, orientação sexual, socioeconômica, política, ambiental, cultural, ética e demais aspectos que compõem o espectro da diversidade humana que singularizam cada pessoa ou cada grupo social[... $]^{8}$.

A tratativa das dimensões das diversidades, fomentadas nas DCN, de modo geral, pode sofrer na sua concretização de notável marginalização, uma vez que as instituições entendam que, ao orientarem o trabalho transversal dos temas, fazemno com pouca ênfase, de forma genérica e superficial, sem aprofundar a formação acadêmica nas políticas públicas, na relação com os movimentos sociais e programas de saúde, mesmo com a intencionalidade de ser inclusiva.

Pensaremadentrarnessestemas dependeprimeiramente de ressignificar a formação médica, fundamentada em um ensino cis-heteronormativo, e ainda entender que cabe à escola médica estimular ações que possam colaborar numa maior abrangência de ensino regrado no compromisso social ${ }^{38}$.

Para ser socialmente referenciada, a escola médica deve ser um espaço onde se problematizem o racismo e a discriminação dos povos originários, o capacitismo, o machismo, a LGBTfobia e todas as formas de opressão, visando à saúde mental e ao bem-estar de docentes, discentes e daqueles(as) que vão participar de forma mais enfática da vida acadêmica a partir dessa inclusão.

As instituições precisam assumir o compromisso de incorporar as temáticas não só nos textos e nas referências bibliográficas, mas também trazer a diversidade de representação para os corpos docente e discente por meio de ações afirmativas, como na constituição das relações acadêmicas com as comunidades e os movimentos sociais. Essa integração deve ser assumida com o compromisso acadêmico de mutualidade desde a construção do marco teórico até a execução das atividades vivenciais, não só ofertando parcerias pautadas no assistencialismo, mas na construção de pontes com o SUS e cenários de práticas que se articulem com as políticas e programas de saúde vigentes com ensino, pesquisa e extensão.

Entretanto, que diversidades, grupos, políticas e programas devem ser trabalhados nesses temas? No Quadro 1, apresentamos algumas dessas políticas/programas nacionais que devemos como educadores(as) ensejar aos(às) estudantes e aos(às) próprios(as) docentes das áreas não relacionadas à APS, SC e CSHS, tendo a compreensão de que, em cada município e estado, essas diretrizes assumem contornos específicos que devem ser incorporados aos currículos locais, como as políticas regionais de saúde que visam ampliar o cuidado com imigrantes e refugiados.

Quadro 1. Políticas e programas públicos de saúde para grupos vulnerabilizados, Brasil, 2020.

\begin{tabular}{|c|c|c|c|}
\hline Política/programa & Objetivo & Ano de publicação & Documento Legal \\
\hline População indígena & $\begin{array}{l}\text { Garantir aos povos indígenas o acesso à atenção integral à } \\
\text { saúde, de acordo com os princípios e as diretrizes do SUS, } \\
\text { contemplando as diversidades social, cultural, geográfica, } \\
\text { histórica e política, de modo a favorecer a superação dos } \\
\text { fatores que tornam essa população mais vulnerável aos } \\
\text { agravos à saúde de maior magnitude e transcendência } \\
\text { entre os brasileiros, reconhecendo a eficácia de sua } \\
\text { medicina e o direito desses povos à sua cultura. }\end{array}$ & 2002 & $\begin{array}{c}\text { Portaria } n^{\circ} 254 \text { de } \\
28 \text { de janeiro }\end{array}$ \\
\hline $\begin{array}{c}\text { População em situação } \\
\text { de rua }\end{array}$ & $\begin{array}{l}\text { Indignação com a violência e a negação de direitos a esta } \\
\text { população. Instituição do conselho técnico. }\end{array}$ & 2009 & $\begin{array}{c}\text { Portaria do } \\
\text { Ministério da } \\
\text { Saúde n } 3 \cdot 305 \text {, de } \\
24 \text { de dezembro }\end{array}$ \\
\hline
\end{tabular}

Continua... 
Quadro 1. Continuação - Políticas e programas públicos de saúde para grupos vulnerabilizados, Brasil, 2020.

\begin{tabular}{|c|c|c|c|}
\hline Política/programa & Objetivo & Ano de publicação & Documento Legal \\
\hline População negra & $\begin{array}{l}\text { Reconhecimento do racismo e da luta contra o racismo } \\
\text { institucional. }\end{array}$ & 2011 & $\begin{array}{l}\text { Portaria } \mathrm{n}^{\circ} 992, \text { de } \\
13 \text { de maio }\end{array}$ \\
\hline População LGBTQI+ & $\begin{array}{l}\text { Discriminação por orientação sexual e identidade de } \\
\text { gênero. }\end{array}$ & 2011 & $\begin{array}{l}\text { Portaria } \mathrm{n}^{\circ} 2.826 \text {, } \\
\text { de } 6 \text { de novembro }\end{array}$ \\
\hline $\begin{array}{l}\text { Pessoas privadas de } \\
\text { liberdade }\end{array}$ & $\begin{array}{l}\text { Inclusão da população penitenciária no SUS, garantindo } \\
\text { que o direito à cidadania se efetive na perspectiva dos } \\
\text { direitos humanos. }\end{array}$ & 2014 & $\begin{array}{c}\text { Portaria } \\
\text { Interministerial } n^{\circ} \\
1 \text {, de } 2 \text { de janeiro }\end{array}$ \\
\hline $\begin{array}{l}\text { Povos do campo, das } \\
\text { florestas e das águas }\end{array}$ & $\begin{array}{l}\text { Reconhecimento de distintos modos de viver e produzir; } \\
\text { redução dos riscos e agravos à saúde decorrentes do } \\
\text { processo de trabalho e das tecnologias agrícolas. }\end{array}$ & 2014 & $\begin{array}{l}\text { Portaria } n^{\circ} 2.311, \\
\text { de } 23 \text { de outubro }\end{array}$ \\
\hline Pessoas com deficiência & $\begin{array}{l}\text { Assegurar e promover, em condições de igualdade, o } \\
\text { exercício dos direitos e das liberdades fundamentais da } \\
\text { pessoa com deficiência, visando à sua inclusão social e } \\
\text { cidadania. }\end{array}$ & 2015 & $\begin{array}{c}\text { Lei } n^{\circ} 13.146 \text {, de } 6 \\
\text { de julho }\end{array}$ \\
\hline Povo cigano & $\begin{array}{l}\text { Promover a saúde integral do povo cigano/romani, } \\
\text { respeitando suas práticas, seus saberes e suas medicinas } \\
\text { tradicionais, priorizando a redução da ciganofobia ou } \\
\text { romafobia e o combate a elas. }\end{array}$ & 2018 & $\begin{array}{l}\text { Portaria } \mathrm{n}^{\circ} \\
\text { 4.384, de } 28 \text { de } \\
\text { dezembro }\end{array}$ \\
\hline
\end{tabular}

Fonte: Elaborado pelos autores.

\section{CONCLUSÃO}

Para além das DCN de 2014, a Resolução nº 569 do Conselho Nacional de Saúde (CNS) 39 apresenta princípios gerais para as diretrizes de todos os cursos da área da saúde. De acordo com essa resolução, as competências necessárias a um(a) egresso(a) do curso da área da saúde, como a Medicina, perpassa a defesa da vida e do SUS, com a redução de iniquidades em saúde ${ }^{16}$ e atendimento às reais necessidades sociais em saúde da população, o que reitera também a responsabilidade social da escola médica.

Para que isso ocorra, o documento aponta para a importância da integração ensino-serviço-gestão-comunidade, entendendo que a formação em saúde está intrinsecamente ligada ao mundo do trabalho e à prática cotidiana, necessitando também valorizar as demandas em saúde da comunidade, especialmente em se tratando de populações historicamente negligenciadas. A resolução menciona também a importância da educação e da comunicação em saúde, que podem ser aprimoradas, por exemplo, com a abordagem da comunicação não violenta e com o compromisso de construção compartilhada de conhecimento, à luz do que é exposto na Política Nacional de Educação Popular em Saúde ${ }^{40}$.

Soma-se aesse cenário o fato de as políticas de acesso ao ensino superior contribuírem para um novo perfil do(a) egresso(a) ${ }^{41}$, inserindo no contexto da educação médica as vivências que esses(as) estudantes têm por serem parte de grupos historicamente invisibilizados. Para além disso, construções fora da sala de aula, como o surgimento de coletivos estudantis auto-organizados, fizeram, durante a pandemia da Covid-19, que as demandas de discussões negligenciadas pela universidade ou colocadas anteriormente apenas como alvo da extensão universitária assumissem destaque no currículo paralelo por meio de seminários virtuais. É esperado que esse movimento repercuta nas salas de aula, pressionando docentes e a gestão acadêmica no sentido de efetivar as expectativas e lacunas de ensino-aprendizado em relação à diversidade, conforme apontam as DCN de 2014.

Assim, como exposto, a pandemia da Covid-19 mostrase como uma oportunidade em que a mídia e a sociedade como um todo olhem para as desigualdades sociais em saúde, considerem a relevância do SUS e enfatizem os múltiplos apontamentos contemporâneos que evidenciam que os projetos pedagógicos e os componentes curriculares dos cursos de Medicina precisam se atualizar e se comprometer com a construção de uma proposta de ensino e cuidado em saúde que valorize a diversidade e a diminuição das iniquidades em saúde. 


\section{AGRADECIMENTOS}

Agradecemos a todos(as) os(as) integrantes dos grupos de trabalho Populações (In)Visibilizadas e Diversidades - da Associação Brasileira de Educação Médica-, Saúde da População Negra e Gênero, Sexualidade, Diversidade e Direitos - ambos da Sociedade Brasileira de Medicina de Família e Comunidade. Agradecemos também a todas as pessoas que de alguma forma apoiam essa luta.

\section{CONTRIBUIÇÃO DOS AUTORES}

Todos(as) os(as) autores(as) contribuíram igualmente na construção do artigo.

\section{CONFLITO DE INTERESSES}

\section{Os(as) autores(as) declaram não haver conflito de} interesses neste estudo.

\section{REFERÊNCIAS}

1. Brasil. Constituição da República Federativa do Brasil. Brasília, DF: Senado Federal; 1988.

2. The Net. Global Consensus for Social Accountability of Medical Schools. Vancouver; 2010. [acesso em 5 ago 2020]. Disponível em: http:// healthsocialaccountability.sites.olt.ubc.ca/files/2011/06/11-06-07-GCSAEnglish-pdf-style.pdf.

3. Boelen C. Global Consensus on the Social Accountability of Medical Schools. Santé Publique, 2011;23(3):247-50.

4. Brasil. Lei n 8.080 , de 19 de setembro de 1990. Lei Orgânica da Saúde. Dispõe sobre as condições para a promoção, proteção e recuperação da saúde, a organização e o funcionamento dos serviços correspondentes e dá outras providências. Brasília; 1990.

5. Lancet T. Redefining vulnerability in the era of COVID-19. Lancet. 2020;395(10230):1089. [acesso em 9 ago 2020]. Disponível em: https:// www.ncbi.nlm.nih.gov/pmc/articles/PMC7270489/pdf/main.pdf.

6. Brasil. Políticas de promoção da equidade em saúde. Brasília: Ministério da Saúde; 2013.

7. Frenk J, Chen L, Bhutta ZA, Cohen J, Crisp N, Evans T, et al. Health professionals for a new century: transforming education to strengthen health systems in an interdependent world. Lancet. 2010;376(4):1923-58.

8. Brasil. Resolução $n^{\circ} 3$, de 20 de Junho de 2014. Diretrizes Curriculares Nacionais do Curso de Graduação em Medicina. Brasília: Ministério da Educação; 2014

9. Oliveira S, Postal E, Afonso D. As escolas médicas e os desafios da formação médica diante da epidemia brasileira da COVID-19: das (in)certezas acadêmicas ao compromisso social. APS em Revista. 2020;2(1):56-60. [acesso em 6 ago 2020] Disponível em: https://aps. emnuvens.com.br/aps/article/view/69.

10. Brasil. Diretrizes Curriculares Nacionais dos Cursos de Graduação em Enfermagem, Medicina e Nutrição. Brasília: Ministério da Educação; 2001.

11. Ferreira MJM, Ribeiro KG, Almeida MM de, Sousa MS de, Ribeiro MTAM, Machado MMT, et al. Novas Diretrizes Curriculares Nacionais para os cursos de Medicina: oportunidades para ressignificar a formação. Interface (Botucatu). 2019;23(1):e170920. doi: 10.1590/interface.170920.

12. Amoretti R. A educação médica diante das necessidades sociais em saúde. RevBrasEduc Med.2020;29(2):136-46. doi: 10.1590/1981-5271v29.2-020.

13. Barros NF. As ciências sociais na educação médica. São Paulo: Hucitec; 2016.

14. Canesqui AM. Sobre a presença das ciências sociais e humanas na saúde pública. SaúdeSoc. 2011;20(1):16-21. doi:10.1590/S0104-12902011000100003.
15. Ritz SA, Beatty K, Ellaway RH. Accounting for social accountability: developing critiques of social accountability within medical education. Educ Health. 2014;27:152-7. [acesso em 5 ago 2020] Disponível em: http:// www.educationforhealth.net/text.asp?2014/27/2/152/143747.

16. Barata RB. Como e por que as desigualdades sociais fazem mal à saúde. Rio de Janeiro: Fiocruz; 2009. [acesso em 6 ago 2020] Disponível em: https:/l static.scielo.org/scielobooks/48z26/pdf/barata-9788575413913.pdf.

17. Brasil. Política Nacional de Saúde Integral da População Negra: uma política do SUS. 2aed. Brasília: Ministério da Saúde; 2013 [acesso emo9 ag02020]; Disponível em: http://bvsms.saude.gov.br/bvs/publicacoes/ politica_nacional_saude integral_populacao.pdf.

18. Raimondi GA, Sousa LP de, Moraes VHA, Afonso DH, Borret RHES, Tourinho FSV, et al. Posicionamento do grupo de trabalho Populações (In) Visibilizadas e Diversidades a Respeito das Violências contra a População Negra e do Racismo Estrutural. RevBrasEduc Med. 2020;44(3):e093. doi: 10.1590/1981-5271v44.3-editorial.

19. Brasil. Política Nacional de Atenção à Saúde dos Povos Indígenas. zaed. Brasília: Ministério da Saúde,Fundação Nacional de Saúde; 2002 [acesso em 9 ago 2020]. Disponível em: https://bvsms.saude.gov.br/bvs/ publicacoes/politica saude indigena.pdf.

20. Ranscombe P. Rural areas at risk during COVID-19 pandemic. Lancet Infect Dis. 2020;20(5):545. doi: 10.1016/S1473-3099(20)30301-7.

21. Worley P. Why we need better rural and remote health, now more than ever. Rural Remote Health. 2020;20:5976. doi:10.22605/RRH5976.

22. Floss M, Franco CM, Malvezzi C, Silva KV, Costa BR, Silva VXL e, et al. A pandemia de COVID-19 em territórios rurais e remotos: perspectiva de médicas e médicos de família e comunidade sobre a atenção primária à saúde. Cad. SaudePublica. 2020;36(7):e00108920. doi: 10.1590/0102$311 \times 00108920$.

23. Greene-Moton E, Minkler M. Cultural competence or cultural humility? Moving beyond the debate. Health PromotPract. 2020;21(1):142-45. doi: 10.1177/1524839919884912.

24. Brasil. Política Nacional de Saúde Integral de Lésbicas, Gays, Bissexuais, Travestis e Transexuais. Brasília: Ministério da Saúde; 2013. [acesso em 6 ago 2020] Disponível em: http://bvsms.saude.gov.br/bvs/publicacoes/ politica_nacional_saude_lesbicas_gays.pdf.

25. Moscheta MD, Fébole DS, Anzolin B. Visibilidade seletiva: a influência da heterossexualidade compulsória nos cuidados em saúde de homens gays e mulheres lésbicas e bissexuais. Saúde Transform Soc. 2016;7(3):71-83.

26. Paulino DB, Rasera EF, Teixeira FB. Discursos sobre o cuidado em saúde de Lésbicas, Gays, Bissexuais, Travestis, Transexuais (LGBT) entre médicas(os) da Estratégia Saúde da Família. Interface (Botucatu). 2019;23:e180279. doi: 10.1590/interface.180279.

27. Wenceslau LD, Fonseca VKT, Dutra LA, Caldeira LG. Um roteiro de entrevista clínica centrada na pessoa para a graduação médica. RevBrasMedFam Comunidade. 2020;15(42):2154. doi: 10.5712/rbmfc14(41)2154.

28. World Health Organization. COVID-19 and the human rights of LGBTI people. United Nations Human Rights; 2020. [acesso em 9 ago 2020]. Disponível em: https://www.ohchr.org/Documents//ssues/LGBT/LGBTIpeople.pdf.

29. Brasil. Política Nacional de Atenção Integral à Saúde da Mulher - princípios e diretrizes. Brasília: Secretaria de Atenção à Saúde, Departamento de Ações Programáticas Estratégicas; 2004. [acesso em 6 ago 2020]. Disponível em: https://bvsms.saude.gov.br/bvs/publicacoes/politica_nac atencao_mulher.pdf.

30. World Health Organization. Integrating gender into the curricula for health professionals. Geneva: WHO; 2006.

31. Brasil. Política Nacional de Atenção Integral à Saúde do Homem. Brasília: Ministério da Saúde; 2008. [acesso em 9 ago 2020]. Disponível em: http://bvsms.saude.gov.br/bvs/publicacoes/politica nacional atencao saude_homem.pdf.

32. Brasil. Diretrizes Nacionais para a Atenção Integral à Saúde de Adolescentes e Jovens na Promoção, Proteção e Recuperação da Saúde. Brasília: Ministério da Saúde; 2010. [acesso em 9 ago 2020]. Disponível em: https://bvsms.saude.gov.br/bvs/publicacoes/diretrizes_nacionais atencao saude adolescentes jovens promocao saude.pdf. 
33. Brasil. Portaria ${ }^{\circ} 2528 / G M$, de 19 de outubro de 2006. Aprova a Política Nacional de Saúde da Pessoa Idosa. Brasília: Ministério da Saúde; 2006 [acesso em 09 ago. 2020]. Disponível em: http://bvsms.saude.gov.br/bvs/ saudelegis/gm/2006/prt2528_19_10_2006.html.

34. Raimondi GA, Abreu YR, Borges IM, Silva GBM, Hattori WT, Paulino DB. Gênero e sexualidade nas escolas médicas federais do Brasil: uma análise de projetos pedagógicos curriculares. RevBrasEduc Med. 2020;44(2):e045. doi: 10.1590/1981-5271v44.2-20190050.

35. Brasil. Política Nacional para Inclusão Social da População em Situação de Rua. Brasília: Ministério da Saúde; 2008. [acesso em 06 ago. 2020] Disponível em: http://www.mpsp.mp.br/portal/page/portal/cao_civel/ acoes_afirmativas/inclusaooutros/aa_diversos/Pol.Nacional-Morad.Rua.pdf.

36. Campos A. População de rua: um olhar da educação interprofissional para os não visíveis. Saúde Soc. 2018;27(4):997-1003. doi: 10.1590/s010412902018180908.

37. Brasil. Política Nacional de Atenção Integral à Saúde das Pessoas Privadas de Liberdade no Sistema Prisional. Brasília: Ministério da Saúde, Ministério da Justiça; 2014. [acesso em 09 ago. 2020]. Disponível em: http://www. as.saude.ms.gov.br/wp-content/uploads/2016/06/Cartilha-PNAISP.pdf.
38. Moretti-Pires RO, Guadagnin LI, Tesser-Júnior ZC, Campos DA, Turatti BO. Preconceito contra diversidade sexual e de gênero entre estudantes de Medicina de $1^{\circ}$ ao $8^{\circ}$ semestre de um curso da Região Sul do Brasil. RevBrasEduc Med. 2020;43(1Supl.1):557-67. doi: 10.1590/1981-5271v43suplemento1-20190076.

39. Conselho Nacional de Saúde. Resolução $n^{\circ} 569$, de 8 de dezembro de 2017. Diário Oficial da União;2018.

40. Brasil. Política Nacional de Educação Popular em Saúde no SUS. Brasília: Ministério da Saúde;2013. [acesso em 05 ago. 2020]. Disponível em: http:// bvsms.saude.gov.br/bvs/saudelegis/gm/2013/prt2761_19_11_2013.html

41. Rego RM, Marques NA, Monteiro PC, Oliveira CLB, Lins NAA, Caldas CEMI. O perfil atual do estudante de Medicina e sua repercussão na vivência do curso. Para Res Med J. 2018;2(1-4):e05. DOI: 10.4322/prmj.2018.005. [acesso em 06 ago. 2020] Disponível em: https://www.prmjournal.org/ article/10.4322/prmj.2018.005/pdf/prmjournal-2-1-4-e05.pdf. 